\title{
Macro ergonomics and health workers during the COVID-19 pandemic
}

\author{
Farahnaz Khajehnasiri $^{\mathrm{a}}$, Vida Zaroushani ${ }^{\mathrm{b}, \mathrm{c}, *}$ and Mohsen Poursadeqiyan ${ }^{\mathrm{d}, \mathrm{e}}$ \\ ${ }^{a}$ Department of Community Medicine, Faculty of Medicine, Tehran University of Medical Sciences, Tehran, Iran \\ ${ }^{\mathrm{b}}$ Social Determinants of Health Research Center, Research Institute for Prevention of Non-Communicable \\ Disease, Qazvin University of Medical Sciences, Qazvin, Iran \\ ${ }^{\mathrm{c}}$ Department of Occupational Health Engineering, Faculty of Health, Qazvin University of Medical Sciences, \\ Qazvin, Iran \\ ${ }^{\mathrm{d}}$ Social Determinants of Health Research Center, Ardabil University of Medical Sciences, Ardabil, Iran \\ ${ }^{\mathrm{e}}$ Department of Occupational Health Engineering, School of Health, Ardabil University of Medical Sciences, \\ Ardabil, Iran
}

Received 7 March 2021

Accepted 18 March 2021

Keywords: COVID-19, occupational health, health workers, ergonomics

Dear Editor-in-Chief,

The COVID-19 pandemic is a disaster all over the world. During this global calamity, the health and safety of workers in the workplace should be considered. Occupational exposure to pathogens is an inherent risk factor of health care workplaces [1]. During the pandemic, the medical staff of hospitals on the frontlines of medical services take the necessary measures to identify patients, isolate them and treat them [2]. The health and safety of these individuals can be enhanced by ergonomics through the redesign and re-evaluation of tasks, environments, and systems. Ergonomics or human factors is "the scientific discipline concerned with the understanding of interactions among humans and other elements of a system, and the profession that applies theory, princi-

*Address for correspondence: Vida Zaroushani, Qazvin University of Medical Sciences, Qazvin, Iran. E-mail: v.zaroushani@ qums.ac.ir. ples, data and methods to design in order to optimize human well-being and overall system performance" (IEA Council, 2014). This science examines working conditions (i.e. physical environment, work organization, psychosocial factors and job design) as well as health and safety behaviors of employees and their participation in programs that can affect their health and system efficiency. It should be noted that the prevention of COVID-19 should not rely on a single infection prevention and control team in the hospital, but should depend on comprehensive programs. The use of human factors and ergonomic knowledge is an effective and profitable framework to address current challenges posed by COVID-19 in hospitals through the utilization of systems approaches (USA; macro ergonomics).

In the USA approach, risk factors are identified and can be controlled. In the study by Gurses et al., risk factors related to patient isolation, physical factors of the environment, technology and tools (screen- 
ing time of admission), processes (patient escort to isolation rooms, communication with patients, isolation, cleaning and reuse of personal protective equipment) were assessed in order to manage risk during a pandemic.

Applying a comprehensive and common strategy based on ergonomic models between different departments of a hospital or between several hospitals is another proposed solution as a response to occupational safety and health problems during the COVID-19 crisis. Sasangohar et al. and Rozman also reported the importance of improving communication and coordination at the system level, including communication between different groups of medical staff [3].

From an ergonomics point of view, it is necessary to determine which abilities and skills should be acquired in a disaster situation. These efforts can improve targeted training strategies in the COVID-19 pandemic, and new training materials should be available to the health care system through the appropriate platforms. The suggestions and concerns of the staff should be heard by the management of the organization, and with their participation in decisions, the current situation can be improved and innovations can emerge in the environment. It is essential that the organization adheres to the use of safety and health protocols to create a sense of security in the hospital staff [4].

Review articles also support the claim that the application of organizational ergonomic models in hospitals can be effective in increasing staff motivation and reducing waste of resources and optimal design of patient care in pandemics [5]. In the current pandemic, the use of documented guidelines on human factors seems necessary.

One of the most important issues in the COVID19 pandemic is the application of efficient methods to protect the workforce against hazards, especially those at the forefront. If this does not happen, the consequences will be catastrophic.

We have to inspect the working conditions using engineering control methods (proper ventilation and separators) and management controls (training of prevention ways, obligation to social distancing, the use of personal protective equipment, establishing sup- port policies to comply with protection, creating a work plan and proper rest, not forcing personnel who have an underlying disease to work in the hospital and consider the workload of personnel).

Ergonomists can identify the points of failure in the hospital environment by direct observation and by studying statistics and documents or inquiries, and by applying an experimental design and evaluating them, new decisions can be made to improve the situation.

As previously mentioned, in a pandemic, with the approach of ergonomics we can identify various risk factors in a hospital and control them. We could also share our valuable ergonomics experiences related to the COVID-19 pandemic by available methods such as webinars. It is furthermore recommended to gather related data and analyze them to create valuable information in order to ensure better future decision making. Moreover, assessing the system by the micro and macro ergonomics approach could be useful to raise work health and job satisfaction.

\section{References}

[1] Wilkason C, Lee C, Sauer LM, Nuzzo J, McClelland A. Assessing and reducing risk to healthcare workers in outbreaks. Health Security. 2020;18(3):205-11.

[2] Quah LJ, Tan BK, Fua TP, Wee CP, Lim CS, Nadarajan G, Zakaria ND, Chan SE, Wan PW, Teo LT, Chua YY. Reorganising the emergency department to manage the COVID-19 outbreak. Int J Emerg Med. 2020;13(1):1-1.

[3] Dennerlein JT, Burke L, Sabbath EL, Williams JA, Peters SE, Wallace L, Karapanos M, Sorensen G. An Integrative Total Worker Health Framework for Keeping Workers Safe and Healthy During the COVID-19 Pandemic. Hum Factors. 2020;62(5):689-96.

[4] Tarzimoghadam S, Zakerian SA. Ergonomics in Healthcare system-Human Factors models: a review. JHSW. 2016;5(4): 87-98.

[5] Sasangohar F, Moats J, Mehta R, Peres SC. Disaster ergonomics: Human factors in COVID-19 pandemic emergency management. Hum Factors. 2020;62(7):1061-8. 